\title{
Prenatally detected, unilateral, high-grade hydronephrosis: Can we predict the natural history?
}

Osama M. Sarhan ${ }^{1}$; Ahmed El Helaly²; Abdul Hakim Al Otay²; Mustafa Al Ghanbar²; Ziad Nakshabandi ${ }^{2}$

${ }^{1}$ Mansoura Urology and Nephrology Centre, Faculty of Medicine, Mansoura University, Mansoura, Egypt; ${ }^{2}$ Pediatric Urology Division, Prince Sultan Military Medical City, Riyadh, Kingdom of Saudi Arabia

Cite as: Can Urol Assoc J 2017 Dec. 22; Epub ahead of print. http://dx.doi.org/10.5489/cuaj.4587

Published online December 22, 2017

\section{$* * *$}

\section{Abstract}

Introduction: Fetal hydronephrosis (HN) occurs in around 5\% of pregnancies and its prognosis depends mainly on the grade of the dilation. We attempted to determine the fate of isolated, unilateral, high-grade $\mathrm{HN}$ in children with antenatal diagnosis, emphasizing the risk factors for progression.

Methods: We retrospectively evaluated 424 children (690 kidney units) with antenatal HN in the period between 2010 and 2014. We included only those patients with isolated high-grade HN (Society for Fetal Urology [SFU] Grade 3 or 4). Patients with bilateral $\mathrm{HN}$ or unilateral $\mathrm{HN}$ associated with dilated ureter or reflux and patients with missed followup were excluded. The prognosis of HN (whether improved, stabilized, or progressed) and the need for surgical intervention in this subset of patients was evaluated.

Results: A total of 44 children (34 boys and 10 girls) were identified. Ultrasounds showed SFU Grade 3 HN in 24 (54\%) and SFU Grade 4 HN in 20 (46\%). After a mean followup of three years (range 1-5), 10 children (23\%) needed surgical intervention; four Grade $3 \mathrm{HN}$ (16\%) and six Grade $4 \mathrm{HN}$ (30\%). The majority of children with differential renal function (DRF) $\geq 40 \%$ (69.5\%) were stable or improved. Five girls (50\%) and five boys (17\%) progressed and required surgical intervention. No patient with a renal pelvis anteroposterior diameter (APD) $<1.5 \mathrm{~cm}$ needed surgical intervention.

Conclusions: Infants with isolated, unilateral, high-grade HN might be managed 
conservatively. Male gender, DRF $\geq 40 \%$, SFU Grade $3 \mathrm{HN}$, and APD $<1.5 \mathrm{~cm}$ were favourable prognostic factors.

\section{Introduction}

Antenatal hydronephrosis (HN) occurs in 1-5\% of all pregnancies. ${ }^{1}$ Once diagnosed; the debate will arise whether it is obstructive or non-obstructive in nature, harmful to the kidney or not, and whether any prenatal or postnatal surgical intervention will be required. $^{2}$

Multiple etiologies of antenatal HN were reported which might include, transient hydronephrosis, pelviureteric junction obstruction, vesicoureteral reflux (VUR), posterior urethral valves or other anomalies. ${ }^{3}$ Exclusion of the fore mentioned disorders define the term isolated antenatal HN. ${ }^{4}$

The exact definition of significant antenatal $\mathrm{HN}$ is evolving. Multiple grading systems and parameters were used to define this entity. However, the society for fetal urology (SFU) grading system and the measurement of antero-posterior diameter (APD) of the renal pelvis were among the commonest utilized. ${ }^{1-3}$

High-grade antenatal HN will usually require extensive evaluation and strict follow-up. This might require the use of continuous antibiotic prophylaxis (CAP), further evaluation, longer follow-up period and they might require surgical intervention, depending on the underlying etiology. ${ }^{4}$ On the other hand, low-grade HN is usually benign in nature and about $50 \%$ of fetuses and infants diagnosed with this entity might show complete resolution on follow-up. ${ }^{5}$

In our study, we tried to study the natural history of isolated high-grade antenatal HN. Our aim was to assign criteria for possible differentiations between cases that deserve observation and those which might necessitate surgical intervention in the hope of aiding the physician in parental counseling and risk stratification.

\section{Methods}

After obtaining the approval of institutional review board at our institute, a retrospective study design was constructed. We identified our patients from the registry of prenatal congenital anomalies in the period from January 2010 to December 2014.

The first postnatal renal ultrasound was obtained during the first week of life in all patients, beyond the first 72 hours. The SFU grading system was used to classify all patients and ultrasound images were again reviewed by two physicians, a pediatric radiologist, and a pediatric urologist, to minimize inter-reviewer variability. If physicians disagreed, the higher grade was taken. Any patient with no available images was excluded from the study. Additionally, measurement of the 
anteroposterior diameter of the renal pelvis (APD), taken at the renal hilum in supine position was recorded.

All patients included in the study were investigated by voiding cystourethrography and cases with vesicoureteral reflux (VUR), posterior urethral valves or ureterocele were excluded.

The first diuretic renography (DR), using mercaptoacetyltriglycine (MAG-3) was performed to all patients 8-12 weeks after birth. A standardized DR was performed universally in all patients. This included adequate IV hydration, MAG-3 was injected using a dose of $0.1 \mathrm{mCi} / \mathrm{kg}$ (minimum $1 \mathrm{mCi}$, maximum $5 \mathrm{mCi}$ ). Differential renal function (DRF) was calculated from the $2^{\text {nd }}$ minute image after drawing tight regions of interest and using a standardized technique for background subtraction. When the entire dilated collecting system was filled with the radiotracer, $1 \mathrm{mg} / \mathrm{kg}$ of furosemide was administered (minimum $1 \mathrm{mg}$, maximum $40 \mathrm{mg}$ ), dynamic images were obtained for $30 \mathrm{~min}$ and T1/2s were calculated post-diuresis. In cases of a flat or rising curve, a $\mathrm{T} 1 / 2$ of $100 \mathrm{~min}$ was assigned. Drainage was classified as good if T1/2 was < 20 min; fair if T1/2 was > 20 min and the drainage curve was descending; or poor if T1/2 could not be counted and there was a rising drainage curve.

We identified 424 patients diagnosed with prenatally detected HN. The first performed postnatal US was used as a base for selection of patients with HN and only those patients with isolated unilateral high-grade HN (SFU grade 3 and 4) were included. We excluded any patient with other anomalies, namely; duplication anomaly, multicystic dysplastic kidney, dilated ureter, VUR, ureterocele, ectopic ureter, urinary bladder diverticulum, thickened bladder wall or posterior urethral valves.

Of 424 patients (690 kidney units), 275 patients (550 kidney units) were excluded due to bilaterality (any grade of HN in the contralateral kidney) . Of the 149 patients with unilateral HN, 105 cases were excluded; 66 with low-grade HN and 39 were found to be associated with other urological anomalies.

The number of urinary tract infections in the included cohort was determined by reviewing urine culture microbiological data. A urinary tract infection was defined as bacterial growth of $10^{5} \mathrm{CFU} / \mathrm{ml}$ when a clean catch technique was utilized and $10^{4}$ $\mathrm{CFU} / \mathrm{ml}$ if catheterized urine sample was used. All patients included in the study were not initially on CAP, and all males included were circumcised at early neonatal period due to religious concern.

During their follow-up, all patients underwent regular renal ultrasonographic examination in intervals that ranged from 1 to 6 months (mean $=3$ months), during the first year of life then ranged from of 3-9 months later on. All patients included in the study had one baseline DR and further studies were performed 6-12 months apart 
during follow-up.

By the end of follow-up period, all included patients were categorized as improved, stabilized or deteriorated based on radiological findings in renal US and DR. Improved cases showed downgrading of HN grade on renal US and/or improved drainage on DR. Stabilized cases had stable HN grade on US and stable DRF $\geq 40 \%$ on DR. Indications for intervention were worsening of $\mathrm{HN}$ for any, deteriorated DRF by more than $10 \%$, DRF below $40 \%$ with an obstructive curve in DR or recurrent febrile UTI. All cases were operated upon within the first year of life.

Statistical analysis was performed using SPSS version 21 (SPSS Inc., Chicago, IL, USA). We used binary and logistic regression analyses. Values are shown as mean \pm SD unless otherwise reported. Kaplan-Meier estimates were used to show the effect of $\mathrm{HN}$ grade, side, and gender on the fate of HN. All tests were 2-sided. A p value of less than 0.05 was considered statistically significant.

\section{Results}

Our study included 44 patients, with isolated unilateral high-grade HN (SFU grade 3 and 4) who met our study criteria for final analysis. Patients' demographics are shown in Table 1. The follow-up period ranged from 2-7 years (mean of 4 years). At the end of follow-up, 10 infants (23\%) had worsening of $\mathrm{HN}$ that required surgical intervention, 4 with grade $3(16 \%)$ and 6 with grade 4 (30\%). Improvement of HN was evident in 12 patients (27\%), while HN continued to be stable in the remaining 22 (50\%).

Using Kaplan-Meier estimates, the effect of gender on progression of HN was statistically significant as it showed that $50 \%$ of girls needed intervention compared to only $17 \%$ of boys, (p-value $=0.043$ ), Figure 1 .

All patients who required surgical intervention had APD of $>15 \mathrm{~mm}$ while, none with APD $\leq 15 \mathrm{~mm}$ needed intervention and that was statistically significant (p-value $=0.001$ ), Figure 2 . The mean APD for deteriorated cases was $25 \mathrm{~mm}$.

Of patients with $\mathrm{DRF} \geq 40 \%$, only $20 \%$ required intervention, whereas those with DRF $<40 \%$, 33.3\% needed intervention and the difference was also statistically significant ( $\mathrm{p}$-value $=0.004)$, Figure 3 .

Urinary tract infections occurred in 4 children (9\%) of our cohort, none of them was initially on CAP, all were grade $4 \mathrm{HN}$ and all needed surgery. The isolated microorganisms were pseudomonas aeruginosa in 2 patients, E.Coli in 1 and klebsiella pneumoniae in another.

Furthermore, all patients who underwent pyeloplasty showed improved HN and non-obstructive curve on DR postoperatively with a mean follow-up period of 2 years 
and none of them developed UTI.

\section{Discussion}

Improvements of the health care facilities and US technology made fetal HN and other genitourinary anomalies easily diagnosed during maternal antenatal screening. In the past, these anomalies were diagnosed later in life when they are symptomatic or complicated. ${ }^{6}$

Hydronephrosis is an anatomical term and is not equivalent to obstruction, and not an indicator of renal function impairment. It may show improvement or spontaneous resolution and no single test can predict future obstruction. ${ }^{7}$ Because of these uncertainties and incomplete understanding of the natural history of this anomaly, many controversies coexist in the way how to manage antenatal HN. ${ }^{8}$ Also, the impact of follow-up protocols and interventions in this type of urinary tract anomalies on the terms of patients' outcome still needs to be more clarified. These controversies exist more with high grades of antenatal $\mathrm{HN}$, as it carries a significant risk of uropathology and renal unit function deterioration. ${ }^{9}$ We aimed to determine which of our cohort of patients with unilateral high-grade $\mathrm{HN}$ required intervention in comparison to those who continued to be followed up conservatively.

In our study and after a mean follow-up period of 48 months, $77 \%$ of patients could be managed conservatively and only $23 \%$ needed surgical intervention. The incidence of surgical intervention was two folds higher in grade $4 \mathrm{HN}$ and this coincides with previous studies. ${ }^{10-12}$

Coelho and colleagues reported that $56 \%$ of infants with SFU grade 4 required surgical intervention whereas only $5 \%$ of SFU grade 3 did. ${ }^{10}$ In a meta-analysis done by Sidu and associates, they showed that isolated antenatal $\mathrm{HN}$ was five times more likely to stabilize if associated with SFU grade $1-2$ or APD $<12 \mathrm{~mm}$ than with SFU grade 3 and $4 .^{11}$ Furthermore, Homsy et al reported that $14 \%$ of antenatal HN SFU grade 3 and $32 \%$ of grade 4 showed further deterioration and hence surgery was indicated. $^{12}$

Meanwhile, Ross et al found that the majority of infants with high-grade HN do not require early surgical intervention. Only $44 \%$ of kidneys with grade $4 \mathrm{HN}$ and $4 \%$ of grade $3 \mathrm{HN}$ underwent early surgery for high-grade obstruction. ${ }^{13}$ In contrast, Chertin et al. have shown that $50 \%$ of children followed conservatively went on to surgery. ${ }^{14}$ However, one must recognize that the criteria for surgical intervention are variable and may be affected by the surgeons' and parents' wishes.

In our study, we investigated the effect of renal pelvic anteroposterior diameter (APD) on the fate of isolated unilateral high-grade antenatal HN. We found that no 
patient with APD less than 15 mm needed intervention while $52 \%$ of patients with APD more than $15 \mathrm{~mm}$ required surgery.

Although APD measurement accuracy is debatable itself, this commonly used ultrasonographic parameters in the follow-up examination of hydronephrotic kidneys is prone to hydration effects, over $10 \%$ change in the maximum AP diameter of the pelvis in the hydronephrotic kidney. However, the pelvic AP diameter at renal hilum is less affected and seems to be independent of hydration status, thus it is more reliable than maximum AP diameter as a follow-up tool in $\mathrm{HN}^{15}$

Masson and associates reported that all patients with APD more than $15 \mathrm{~mm}$ went for surgery, but all had obstructed curve on diuretic renography. ${ }^{16}$ Of 178 neonates, Sharifian et al in their study reported 42 patients (23\%) required surgery. The area under the curve for APD to predict the need for surgery was 0.925 with an APD cutoff of $15 \mathrm{~mm}$. The diagnostic value of APD for determining the need for surgery was determined by sensitivity and specificity of $95.2 \%$ and $73.5 \%$, respectively. ${ }^{17}$

Aubin et al investigated the prenatal APD effect and concluded that antenatal HN SFU grades 3 or 4, and APD $9 \mathrm{~mm}$ or greater, on third trimester US, were associated with an increased risk of surgical intervention in the postnatal period. Patients with an SFU grade of 4 progressed to intervention at the fastest rate. ${ }^{18}$

Kim and associates concluded that an APD cutoff of 5, 8, and $10 \mathrm{~mm}$ during the second, early third, and late third trimesters, respectively, is more specific in predicting the need for postnatal surgical intervention in the Korean population. ${ }^{19}$

In our study, most infants with DRF $\geq 40 \%$ were improved or stabilized while in those with DRF below 40\%, one-third required surgical intervention. Chertin et al concluded that conservative management is appropriate for infants with DRF exceeding $40 \%$ even with an obstructive pattern on DR. ${ }^{20}$ Multiple authors go with conservative management in this group of patients but recommend a serial US and repeated DR if there is persistent or progressive HN or parenchymal thinning. ${ }^{21,22}$

We also investigated the effect of gender on the fate of high-grade $\mathrm{HN}$ in our cohort. We found that $50 \%$ of the females and only $17 \%$ of males deteriorated and required intervention and that was statistically significant. We could not find in the literature any effect of gender on the natural history of antenatal HN.

That is may be due to the fact that male fetuses have larger renal pelvic APD and smaller bladder volumes than females. This gender difference in morphology indicates different urinary tract development between male and female fetuses, which likely accounts for gender difference in the urinary tract anomalies detected during neonatal period. ${ }^{23}$

The incidence of UTI among our patients was $9 \%$. No patient with grade 3 
antenatal HN developed UTI, only those with grade 4 had this augmented risk. Of note, UTI events may be underestimated as recording events were dependent on family physician reports, symptoms of UTI and no routine urine cultures were conducted to most of our cohort unless symptomatic. Coelho and colleagues reported that, in a cohort of prenatal HN, the incidence of UTI during follow-up was higher among infants with moderate/severe renal pelvic dilatation (20\%) than among patients with mild dilatation (7.8\%). ${ }^{10}$

Braga et al reported the results of multiple meta-analyses that showed UTI was higher in cases with high-grade antenatal HN than low grade. ${ }^{24}$ It showed the pooled UTI rates in patients with low-grade antenatal HN were similar regardless of CAP status. ${ }^{4,26,27}$ In the high-grade HN group, infants on CAP had a significantly lower UTI rate versus those not on CAP. For this most of them recommended CAP for patients with high-grade antenatal HN. ${ }^{28,29}$ Of note, this overall rate of UTI in most of the studies, regardless multiple other parameters, like gender or VUR, which may affect results interpretation considerably.

American Urological Association Update Series on Prenatal Diagnosis of Urological Disease, 2009, stated that, although CAP is generally recommended for severe (grades III and IV) HN, the practice is not evidence based. ${ }^{28}$ Multiple recent studies showed that there is recovery of kidney function even in cases with delayed surgery in patients with high-grade antenatal $\mathrm{HN}$ and with evidence of obstruction. ${ }^{4}$

Limitations of our study include that this is a retrospective study with its inherited limitation in a fairly small number of patients with highly selective inclusion criteria. No biomarkers for obstruction were involved and the long-term outcome was not addressed in the study. However, future prospective well-designed studies are encouraged to answer our research question about the dilemma of high-grade HN.

\section{Conclusion}

Patients with isolated high-grade antenatal HN still have a good chance for conservative management and we can avoid unnecessary surgery without endangering the kidney function. For parental counseling, male gender, SFU grade 3, DRF more than $40 \%$ and APD less than $15 \mathrm{~mm}$, all are favorable prognostic factors. Close observation is a must especially in the first year of life. 


\section{References}

1. Lee RS, Cendron M, Kinnamon DD, Nguyen HT. Antenatal hydronephrosis as a predictor of postnatal outcome: a meta-analysis. Pediatrics 2006; 118: 586-93.

2. Riccabona M. Assessment and management of newborn hydronephrosis. World $J$ Urol. 2004; 22:73-8.

3. Mallik M, Watson AR. Antenatally detected urinary tract abnormalities: more detection but less action. Ped Nephrol 2008; 23:897-904.

4. Cheng AM, Phan V, Geary DF, Rosenblum ND. Outcome of isolated antenatal hydronephrosis. Arch Pediatr Adolesc Med. 2004; 158:38-40.

5. Barbosa JA, Chow JS, Benson CB, et al. Postnatal longitudinal evaluation of children diagnosed with prenatal hydronephrosis: insights in natural history and referral pattern. Prenat Diagn. 2012; 32:1242-9.

6. Yang Y Hou Y, Niu ZB, Wang CL. Long-term follow-up and management of prenatally detected, isolated hydronephrosis. J Pediatr Surg. 2010; 45:1701-6.

7. Hafez AT, McLorie G, Bagli D, Khoury A. Analysis of trends on serial ultrasound for high grade neonatal hydronephrosis. J Urol. 2002; 168:1518-21.

8. Shokeir AA, Nijman RJ. Antenatal hydronephrosis: changing concepts in diagnosis and subsequent management. BJU Int. 2000; 85:987-94.

9. Karakurt N, Beşbaş N, Bozacı AC, et al. Antenatal hydronephrosis: a single center's experience and follow-up strategies. Turk J Pediatr. 2015; 57:560-5.

10. Coelho GM, Bouzada MC, Pereira AK, et al. Outcome of isolated antenatal hydronephrosis: a prospective cohort study. Pediatr Nephrol. 2007; 22:1727-34.

11. Sidhu G, Beyene J, Rosenblum ND. Outcome of isolated antenatal hydronephrosis: a systematic review and meta-analysis. Pediatr Nephrol. 2006; 21:218-24.

12. Homsy YL, Saad F, Laberge I, et al. Transitional hydronephrosis of the newborn and infant. J Urol. 1990; 144:579-83.

13. Ross SS1, Kardos S, Krill A, Bourland J. Observation of infants with SFU grades 3-4 hydronephrosis: worsening drainage with serial diuresis renography indicates surgical intervention and helps prevent loss of renal function. J Pediatr Urol. 2011; 7:266-71.

14. Chertin B, Pollack A, Koulikov D, et al. Does renal function remain stable after puberty in children with prenatal hydronephrosis and improved renal function after pyeloplasty? J Urol. 2009; 182:1845-8.

15. Cost NG, Prieto JC and Wilcox DT. Screening ultrasound in follow-up after pediatric pyeloplasty. Urology. 2010; 76:175-9. 
16. Masson P, De Luca G, Tapia N, et al. Postnatal investigation and outcome of isolated fetal renal pelvis dilatation. Arch Pediatr. 2009; 16:1103-10.

17. Sharifian M, Esfandiar N1, Mohkam M, et al. Diagnostic accuracy of renal pelvic dilatation in determining outcome of congenital hydronephrosis. Iran J Kidney Dis. 2014; 8:26-30.

18. St Aubin M, Willihnganz-Lawson K, Varda BK, et al. Society for Fetal Urology recommendations for postnatal evaluation of prenatal hydronephrosis - will fewer voiding cystourethrograms lead to more urinary tract infections? J Urol. 2013; 190:1456-61.

19. Kim HJ, Jung HJ, Lee HY, et al. Diagnostic value of anteroposterior diameter of fetal renal pelvis during second and third trimesters in predicting postnatal surgery among Korean population: useful information for antenatal counseling. Urology 2012; 79:1132-7.

20. Chertin B, Pollack A, Koulikov D, et al. Conservative treatment of ureteropelvic junction obstruction in children with antenatal diagnosis of hydronephrosis: lessons learned after 16 years of follow-up. Eur Urol. 2006; 49:734-8.

21. Ransley PG, Dhillon HK, Gordon I, et al. The postnatal management of hydronephrosis diagnosed by prenatal ultrasound. J Urol. 1990; 144:584-7.

22. Areses Trapote R, Urbieta Garagorri MA, Ubetagoyena Arrieta M, et al. Severe primary congenital unilateral hydronephrosis. A review of 98 cases. An Pediatr (Barc). 2006; 64:11-20.

23. Bassanese G, Travan L, D'Ottavio G, et al. Prenatal anteroposterior pelvic diameter cutoffs for postnatal referral for isolated pyelectasis and hydronephrosis: more is not always better. J Urol. 2013; 190:1858-63.

24. Braga LH, Mijovic H, Farrokhyar F, et al. Antibiotic prophylaxis for urinary tract infections in antenatal hydronephrosis. Pediatrics. 2013; 131:e251-61.

25. Brophy MM, Austin PF, Yan Y, Coplen DE. Vesicoureteral reflux and clinical outcomes in infants with prenatally detected hydronephrosis. J Urol. 2002; 168:1716-9.

26. Wollenberg A, Neuhaus TJ, Willi UV, Wisser J. Outcome of fetal renal pelvic dilatation diagnosed during the third trimester. Ultrasound Obstet Gynecol. 2005; 25:483-8.

27. De Kort EH, Bambang Oetomo S, Zegers SH. The long-term outcome of antenatal hydronephrosis up to 15 millimetres justifies a noninvasive postnatal follow-up. Acta Paediatr. 2008; 97:708-13.

28. Estrada CR, Peters CA, Retik AB, Nguyen HT. Vesicoureteral reflux and urinary tract infection in children with a history of prenatal hydronephrosis—should 
voiding cystourethrography be performed in cases of postnatally persistent grade II hydronephrosis? J Urol. 2009; 181:801-6.

29. Roth CC, Hubanks JM, Bright BC, et al. Occurrence of urinary tract infection in children with significant upper urinary tract obstruction. Urology. 2009; 73:74-8. 


\section{Figures and Tables}

Fig.1. Kaplan-Meier curve showing incidence of progression of hydronephrosis stratifying by gender.

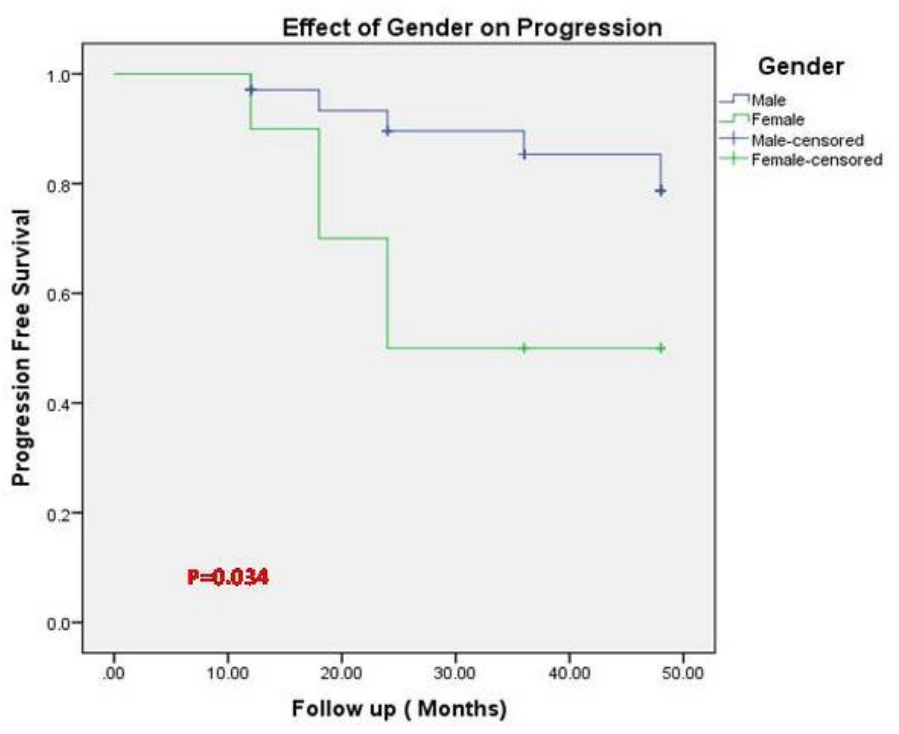

Fig. 2. Kaplan-Meier curve showing incidence of progression of hydronephrosis stratifying by anteroposterior diameter.

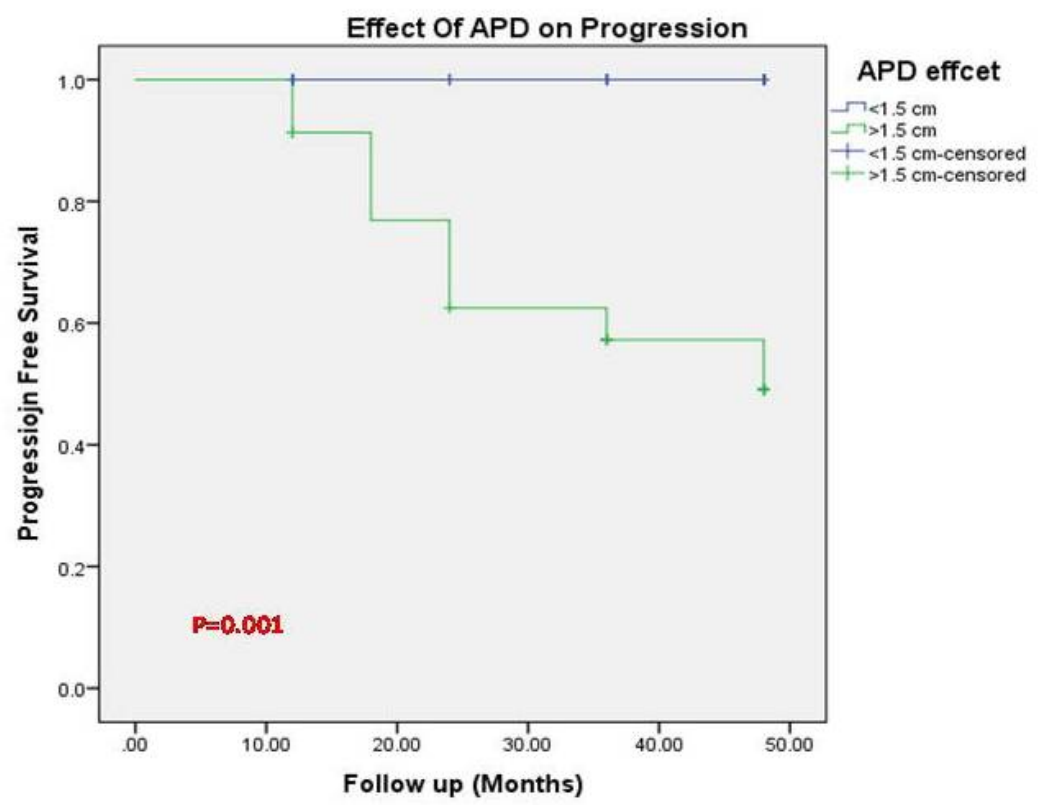


Fig. 3 Kaplan-Meier curve showing incidence of progression of hydronephrosis stratifying by differential renal function.

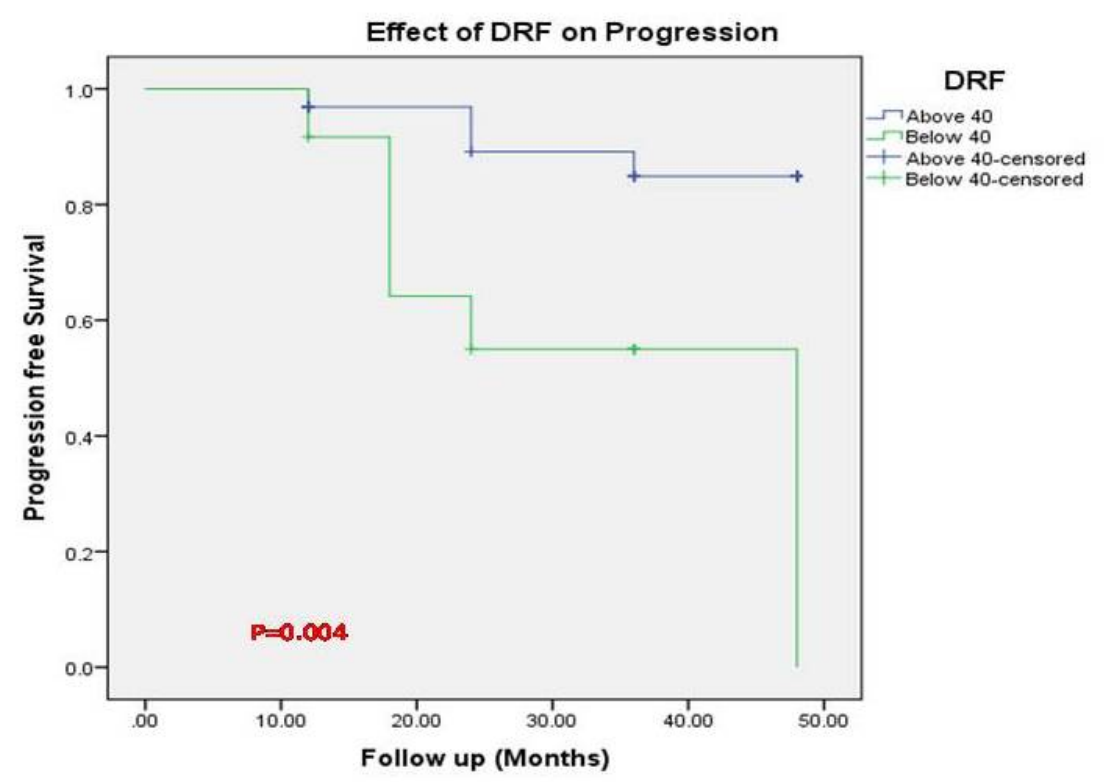

\begin{tabular}{|l|c|c|c|}
\hline \multicolumn{2}{|l|}{ Table 1. Patient demographics } & $\mathbf{n = 4 4}$ & $\mathbf{\%}$ \\
\hline \multicolumn{2}{|l|}{ Unilateral isolated high-Grade HN } & 34 & 77 \\
\hline \multirow{2}{*}{ Gender } & Male & 10 & 23 \\
\cline { 2 - 4 } & Female & 14 & 32 \\
\hline \multirow{2}{*}{ Laterality } & Right & 30 & 68 \\
\cline { 2 - 4 } & Left & 24 & 54.5 \\
\hline \multirow{2}{*}{ SFU } & Grade3 & 20 & 45.5 \\
\cline { 2 - 4 } & Grade 4 & 19 & 43 \\
\hline \multirow{2}{*}{ APD } & $>15 \mathrm{~mm}$ & 25 & 57 \\
\cline { 2 - 4 } & $\leq 15 \mathrm{~mm}$ & 35 & 79.5 \\
\hline \multirow{2}{*}{ DRF } & $\geq 40 \%$ & 9 & 20.5 \\
\cline { 2 - 4 } & $<40 \%$ & 35 & \\
\hline
\end{tabular}

APD: anteroposterior diameter; DRF: differential renal function; HN: hydronephrosis; SFU: Society for Fetal Urology. 